\title{
Preliminary Results of Total Hip Arthroplasty in Subjects at Risk for Dislocation Using a Novel Modular Cementless Dual-Mobility Cup. A Single-Center Prospective Study
}

\author{
Giuseppe Solarino, Filippo Simone *(1), Antonello Panella, Arianna Carlet, Flavia Riefoli and Biagio Moretti \\ Department of Basic Medical Sciences, Neuroscience and Organs of Sense, School of Medicine, AOU Policlinico \\ Consorziale, Università di Bari “Aldo Moro", 70124 Bari, Italy; giuseppe.solarino@uniba.it (G.S.); \\ apanella77@hotmail.com (A.P.); ari.carlet@gmail.com (A.C.); flaviaok91@gmail.com (F.R.); \\ biagio.moretti@uniba.it (B.M.) \\ * Correspondence: filsimo1993991@gmail.com
}

Citation: Solarino, G.; Simone, F.; Panella, A.; Carlet, A.; Riefoli, F.; Moretti, B. Preliminary Results of Total Hip Arthroplasty in Subjects at Risk for Dislocation Using a Novel Modular Cementless Dual-Mobility Cup. A Single-Center Prospective Study. Prosthesis 2021, 3, 53-60. https: / / doi.org/10.3390/ prosthesis 3010006

Received: 20 December 2020 Accepted: 25 January 2021 Published: 1 February 2021

Publisher's Note: MDPI stays neutral with regard to jurisdictional claims in published maps and institutional affiliations.

Copyright: (C) 2021 by the authors Licensee MDPI, Basel, Switzerland. This article is an open access article distributed under the terms and conditions of the Creative Commons Attribution (CC BY) license (https:/ / creativecommons.org/licenses/by/ $4.0 /)$.

\begin{abstract}
Nowadays total hip arthroplasty (THA) is widely considered the operation of the century in orthopedic and traumatological fields. Despite this fact, instability and dislocation after THA are a common reason for revision surgery. The purpose of this prospective study is to evaluate the preliminary clinical and radiological results of a novel dual mobility cup. We evaluated 32 consecutive cases of patients who underwent THA using a novel dual mobility cup-with holes in the cup, a modular metallic inlay and a crosslinked polyethylene. All of them were considered at risk of instability of the implant due to primary or concomitant diagnosis. The preoperative Harris hip score was 54.7 on average. At a minimum 2 years follow-up, the mean HHS raised up to a mean value of 88.4; this improvement was statistically significant $(p<0.0001)$. None of the patients enrolled in this study sustained a postoperative dislocation at a mean follow-up of 39.6 months. Furthermore, no patients sustained modular liner dissociation or an intraprosthetic dislocation. As such, survivorship free from dislocation was $100 \%$ at both 2 and 5 years. This study demonstrates that the modular dual mobility (DM) socket provides dual articulation, larger jump distance, and greater range of motion before impingement, which significantly reduce the rate of dislocation.
\end{abstract}

Keywords: hip; total hip arthroplasty; dislocation; instability; dual mobility

\section{Introduction}

In the 1960s, hip replacement revolutionized management of patients affected by hip osteoarthritis, and nowadays total hip arthroplasty (THA) is widely considered one of the most successful procedures producing excellent outcomes, being named the operation of the century in orthopaedical and traumatological fields. Instability and dislocation after THA are a common reason for revision surgery and therefore they are perceived as a serious complication by both surgeons and patients [1].

Instability following THA is a multi-factorial event, with different etiologies, and eventually several categories of risk factors are recognized, related to the patients and/or to the surgeons' choice: primary diagnosis such as femoral neck fracture and post-traumatic arthritis, secondary diagnosis at the neighbor joints such as spino-pelvic imbalance, neurological and psychiatric disorders such as Parkinson's disease, epilepsy, and schizophrenia, implant orientation and design, and laxity of the surrounding soft tissues [2-4].

The concept of dual mobility (DM) was thought up in the 1970s and combined two articulations: one large and one smaller by a recruitment phenomenon increasing the jumps distance and so decreasing dislocation forces, with advantages of large head without adverse effects. Use of first-generation DM was limited worldwide due to concern regarding the fact that the cup, to avoid the flowing of polyethylene (PE) debris and among the plugs of the metallic shell, did not have holes for implementation of fixation with 
additional screws whether required; such a design also did not allow to check for the proper seating of the cup into the reamed acetabulum. Furthermore, wear of the PE could lead to macrophagic reaction and consequent periprosthetic osteolysis and mechanical failure $[5,6]$.

The scope of this prospective single-center study is to report on the preliminary outcomes achieved with the use of a novel dual mobility cup-with holes and macrostructures on the back of the cup, in order to have better mechanical support, with a modular metallic inlay and using crosslinked PE-implanted in subjects at risk for luxation of the THA. To our knowledge, there is no paper in English that describes the system proposed.

\section{Material and Methods}

We prospectively evaluated the records of 32 consecutive cases of patients who underwent primary THR using a novel DM acetabular system; exclusion criteria included patients with less than two-year minimum follow-up and patients with active or previous hip joint infection. Study protocol was in accordance with the Declaration of Helsinki for human research. For each patient, the complete medical history was collected. The cohort of patients were composed by 17 women and 14 men. One female patient underwent bilateral THA consecutively, not simultaneously. The mean age was 60.8 years (range 23-84) and the mean follow-up was 39.6 months (24-50). The right hip was operated in 19 cases, the left in 13.

Preoperatively clinical assessment was done using the Harris hip score (HHS): the preoperative HHS was 54.7 on average (range, 47-64); for radiological evaluation an anteroposterior pelvic radiograph was obtained to estimate the predicted cup size. Templating was performed by two fellowship-trained orthopedic surgeons. The main diagnosis that had led to the operations was: 14 primary hip osteoarthritis, 11 intracapsular fracture of the femoral neck, 6 post-traumatic osteoarthritis after internal fixation of acetabular fracture and 1 conversion to THA of a failed osteosynthesis with an intramedullary nail due to intertrochanteric fracture.

Informed consent was obtained from all patients. Concomitant diseases, considered as risk factors for THA instability, were registered as follows: Parkinson disease in 6 cases, schizophrenia in 2 case, epilepsy in 4 cases, hip-spine syndrome in 10 cases.

All patients were intravenously administered an antibiotic prophylaxis using $2 \mathrm{~g}$ of Cefazolin during the operation and $1 \mathrm{~g}$ every $6 \mathrm{~h}$ for a total of 3 postoperative doses. In general anesthesia or combined spinal-epidural anesthetic all the THAs were implanted using the direct lateral approach (with patient laying in lateral decubitus position on the contralateral unaffected side) in a conventional turbulent flow theatre. Operations were done by 3 senior orthopedic surgeons; none was performed by a trainee as first surgeon, even under supervision

The acetabular component (G7 Dual Mobility Acetabular system ${ }^{\circledR}$, Zimmer-Biomet, Warsaw, Indiana, USA) is a 3D porous titanium alloy acetabular cup with a mean pore size of 475 microns, $70 \%$ porosity, and a coefficient of friction of 1.25 . A multi-hole design for the acetabular cup was used in all cases (Figure 1): it was press-fit after progressive reaming of the acetabular cavity, with an intended inclination angle at $40^{\circ}-50^{\circ}$ and an intended anteversion at $10^{\circ}-20^{\circ}$. The diameter of the acetabular component ranged from $48 \mathrm{~mm}$ to $58 \mathrm{~mm}$ as follows: size 50 in 10 cases, size 52 in 8 cases, size 54 in 5 case, size 56 in 4 cases, size 48 in 3 cases, and size 58 in 2 cases. Two or more additional acetabular screws were used in 14 cases to implement fixation whether the press-fit stability was in question, or where the bone quality was not optimal, and according to the preference of the surgeon; in these cases protrusion of the screw head must obviously be avoided to allow for the proper seating of the metallic modular liner. 


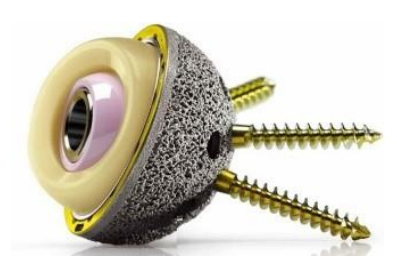

Figure 1. G7 Dual Mobility Acetabular system ${ }^{\circledR}$ is a cup made of a titanium-alloy core, with microporous outer surface and multiple holes to improve fixation with additional screws if needed. A polar pilot hole allows for proper insertion of the chrome-cobalt definitive liner; it is coupled with a large head in highly-crosslinked polyethylene. The femoral head is compressed into it intraoperatively.

The chrome-cobalt definitive liner implant was manually introduced, after ensuring that the interior of the shell and all tapers were dry. A polar pilot hole allows the taper interface to properly be aligned for seating of the definitive metal liner, that should be impacted with several moderate mallet strikes. Thereafter it is compulsory to visually verify whether the ring is fully seated against the face of the liner: when properly seated, the liner will sit flush with the face of the shell.

Two different types of crosslinked PE were used: ArCom $\mathrm{XL}^{\circledR}$ used in 9 cases, and antioxidant vitamin-E infused $\mathrm{E} 1^{\circledR}$ in 23 cases.

On the femoral side in all cases we implanted cementless stems: 19 Taperloc, 9 CLS, 3 Conus and 1 Arcos (all manufactured by Zimmer-Biomet, Warsaw, Indiana, USA). Progressive broaches were manually used to prepare the femoral medullary canal until resistance against the inner cortex was felt; the chosen stem was then inserted by guiding it with its appropriate device, aiming to obtain approximately $10-20^{\circ}$ of anteversion.

Before closure in layers, the stability of the hip implant was assessed examining the primary arc range of motion. Perioperative care was the same for all patients: thromboembolic prophylaxis with low molecular weight heparin was administered for five weeks; during the same period of time patients used compression stockings. Passive motion exercises with the assistance of a therapist started immediately after the operation; patients were free to walk with two supports after 3 days for about 6 weeks and thereafter full weight-bearing was usually allowed.

\section{Results}

All statistical analyses were performed using MedCalc Version 18.2.1. Quantitative variables were analyzed using a Student's t-test. A $p$ value of less than 0.05 was considered statistically significant. A clinical and radiographic evaluation was performed at 1, 3, 6, 12 months postoperatively and annually thereafter. All patients completed the HHS pre-operatively and at a minimum of two-years post-operatively. At the last follow-up the Harris Hip Score (HHS) was submitted to the patients. All patients declared that they were satisfied with the results of surgery, showed clinical improvement, and walked normally without any help at 3 months after surgery, the mean HHS being raised up to a mean value of 88.4 (range, $84-93$ ). This improvement was statistically significant $(p<0.0001)$. No clear relationship between HHS, gender, age, diagnosis, and prosthetic components dimension were seen in this study. Radiologically, any sign of tilting/loosening/migration of the acetabular component was documented. All eventual local complications, as intraoperative periprosthetic fracture, periprosthetic infection, dislocation, breakages of the polyethylene and/or of the head, dissociation within the prosthesis, were recorded. Any eventual clicking and/or squeaking was also recorded. Failure was defined when a subjective feeling of instability was referred and/or whether a revision surgery for any cause was needed, because this is a firm, objective endpoint.

None of the patients enrolled in this study sustained a postoperative dislocation at a mean follow-up of 39.6 months. Furthermore, no patients sustained modular liner dissociation or an intraprosthetic dislocation. As such, survivorship free from dislocation was $100 \%$ at both 2 and 5 years. 
One patient (3.12\%) experienced a complication and he underwent a superficial irrigation and debridement for a superficial wound infection without deep penetration at 1 month postoperatively.

\section{Discussion}

Dislocation after THA has become a significant problem, surpassing aseptic loosening; DM implants are currently selected particularly often for patients at high risk of postoperative instability.

Fractures of the femoral neck in elderly population are very common in orthopedic practice and THA is considered the best option of treatment. The size of the femoral head is an important factor that influences the outcome of THA: implants with a $28 \mathrm{~mm}$ femoral head are more prone to dislocate than implants with a $32 \mathrm{~mm}$ head or larger. Large metal-onmetal bearings are no longer used and ceramic-on-ceramic bearings have proven long-term satisfactory outcomes, but some specific complications such as implant breakages and noise have arisen; therefore nowadays PE is still considered the best tribological option for THA in most of the patients worldwide. Obviously, a large head coupled to a polyethylene inlay can lead to more wear, osteolysis, and failure of the implant. Furthermore, patients with THA done for femoral fracture are more prone to dislocation because of the attempt to regain the complete range of motion the patient had before the fall and due to the sarcopenia of the surrounding muscular tissues in the elderly, that causes laxity of the artificial joint. The dual mobility concept is a PE insert, locked on the femoral ball-head, which moves about freely in a press-fit metallic shell, in fact realizing a compromise that conciliates two advantages: low friction (i.e., less wear) and large heads (i.e., less dislocation) $[7,8]$. In our series we do not report any dislocation of the 11 implants done for femoral neck fracture, thus demonstrating that the use of DM cups in the treatment of displaced fractures of the femoral neck appears safer in terms of the criteria of postoperative dislocation when compared to conventional cups. In their systematic review including 59 articles and 17,908 THAs, De Martino et al. [9] reported the rate of dislocation following the use of DM acetabular components: the mean rate of dislocation was $0.9 \%$ in the primary THA group and they concluded that the use of DM acetabular components is effective in minimizing the risk of instability after THA. Eventually this benefit must be balanced against continuing concerns about the additional modularity: our little sample demonstrates no negative effects in a short follow-up period.

Some patients in our series were also affected by altered mental status or neuromuscular diseases; some authors have stated that psychological factors and mental status, such as depression, anxiety, schizophrenia, or dementia can affect outcome in primary total hip (and knee) replacement. These comorbidities determine the prevalence and are firmly associated with increased odds of adverse events, suggesting that psychological support, together with specific implants, should be rigorous steps to follow for gaining subjective and objective satisfactory clinical results [10-12]. Dual mobility hip replacement has been also proposed in ambulatory patients with cerebral palsy in the more severe Gross Motor Function Classification System stages. [13]

Figure 2 shows the case of a 54 years old male who was admitted to our hospital due to a displaced fracture of his left femoral neck and was in treatment since several years for depression, treated with the G7 modular cementless DM cup with 2 screws and a Taperloc stem on the femoral side. 


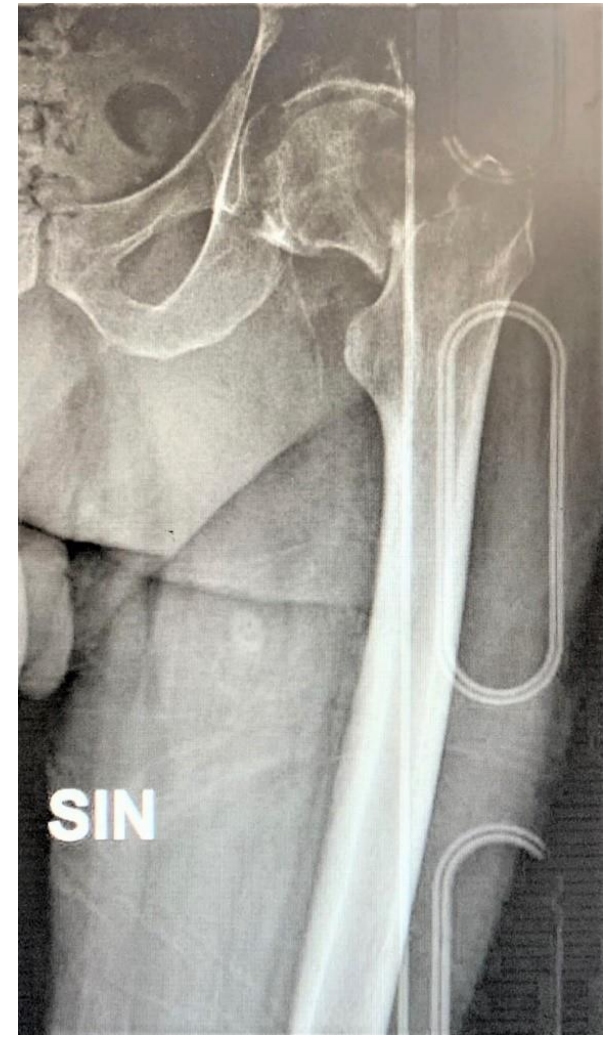

(a)

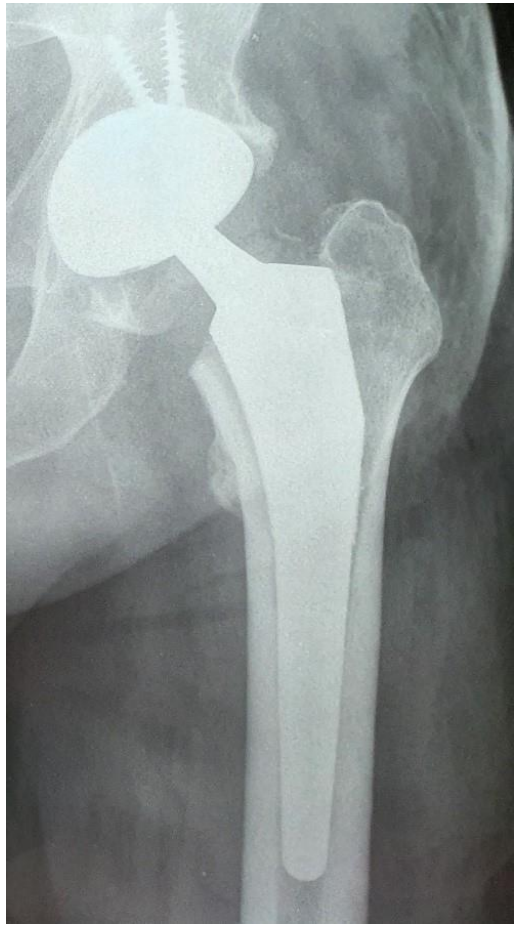

(b)

Figure 2. Displaced fracture of left femoral neck in a 54 years old male (a); dual mobility (DM) modular cup with screw and cementless stem (b).

Intertrochanteric fracture, mainly due to osteoporosis or sometimes severe trauma, is one of the most frequent fractures in orthopedic surgery and is usually treated by internal fixation. Depending on several factors, a small group of patients have complications including nonunion, malunion, avascular necrosis, or failure of the device; thus the incidence of failure of internal fixation should be encountered in a certain percentage and an option currently used is the conversion to THA.

Hip arthroplasty after failure of internal fixation is challenging, and may be complicated by dislocation in a relatively short postoperative period, because these patients were nonambulatory for a while and senile muscular hypotrophy would not be improved except with ambulation; an eventual further damage of the hip stabilizers may also be added when removing from the femur the failed internal fixation devices, sometimes with broken screws [14-17]. Archibeck et al. [18] reported that 12 patients of their 102 who had undergone THA after failed internal fixation of proximal femoral fractures had early surgical complications related to the procedure, dislocations being 5 of them, while in their multi-center study Pui et al. claimed rates of postoperative dislocations of greater than $15 \%$ [19]. Conversion in THA after failed internal fixation of pelvic or femoral fractures is nowadays a clear indication for DM implants; in our 7 cases (6 posttraumatic osteoarthritis and 1 conversion) we do not report any dislocation of the prosthesis.

Concurrent hip and spine pathologies can alter the biomechanics of spinopelvic mobility in primary THA, and a too high THA dislocation rate has been demonstrated among THA patients with concurrent spinopelvic malalignment. The risk of dislocation increases especially within the first six months in patients who had previously undergone a lumbar fusion involving the sacrum and in patients affected by a sagittal imbalance due to concomitant degenerative pathology of the hip and lumbosacral spine ("hip-spine syndrome") and therefore surgeons should take care with component positioning and must consider modular implants to adapt the prosthesis to the morphometric differences of 
patients, to allow better accuracy in restoring the anatomy and biomechanics of hip joint. In fact, in the above-mentioned scenarios, the degree of lumbar spine lordosis cannot change to allow the pelvis to tilt so that the range of movement demanded from the replaced hip is greater than that required in a normal THA and higher stability implants, such as DM components, must be considered in this high-risk cohort [20-26].

Figure 3 shows the case of a 68 years old female, who was referred to our unit due to persistent low back pain for vertebral steno-instability and consequent sagittal imbalance to undergo spinal lumbo-sacral fusion; clinical and radiological examinations revealed a concomitant severe osteoarthritis in both her hips that was treated with bilateral THA with the G7 modular cementless DM cup. After surgical treatment in the hips, low back pain improved dramatically, and the patient did not need any surgery in her spine.

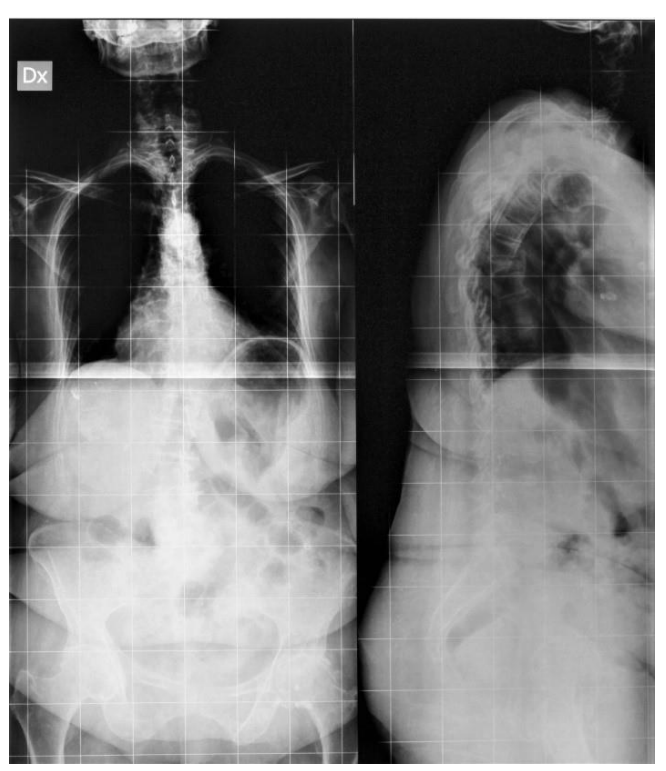

(a)

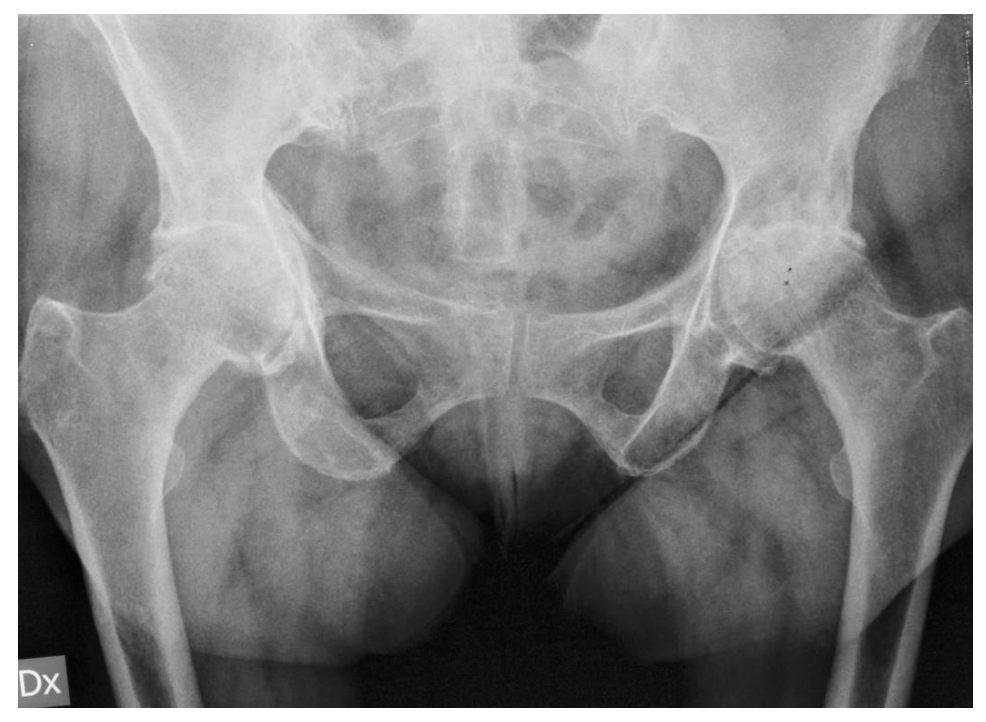

(b)

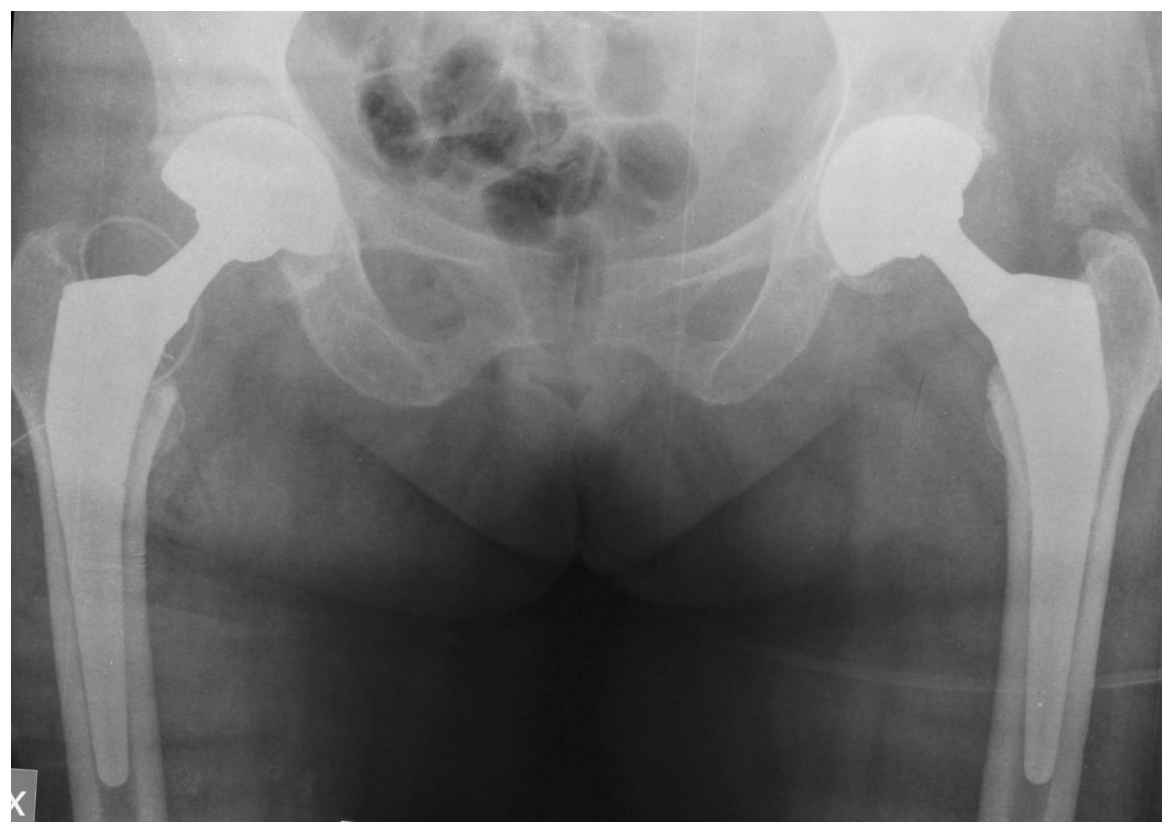

(c)

Figure 3. Steno-instability and consequent sagittal imbalance oh the spine (a); severe bilateral hip osteoarthritis (b); staged bilateral total hip replacements using DM modular cups and cementless stems (c). 
In conclusion, despite decades of investigating improvements in implant design and surgical technique, dislocation following primary THA remains a concern. Our study confirms, using a novel modular acetabular component, that DM implants can avoid this complication, even in patients considered at risk.

Our study has some limitations: first, it is a preliminary report of a small sample size, in the absence of a control group, and with a short follow-up. However, this has ensured that all the patients were eligible at the final follow-up. Second, we have not measured biomechanical parameters in the coronal plane such as vertical and horizontal femoral offset, and abduction of the cup. Finally, despite the liner is in CoCr alloy and the shell is in titanium alloy, we have not monitored metal ion levels.

In conclusion, despite the above-mentioned limitations, our prospective single center study shows that the modular DM socket provides dual articulation, larger jump distance, and greater range of motion before impingement, which significantly reduce the rate of dislocation. Further studies with large cohorts are needed to demonstrate the superiority of this implant compared to conventional ones even at longer follow-up.

Author Contributions: G.S. and F.S. equally conceived and designed the article and drafted the manuscript; A.C. and F.R. collected the data; A.P. coordinated the study; B.M. critically revised the manuscript. All authors have read and agreed to the published version of the manuscript.

Funding: This research received no external funding.

Institutional Review Board Statement: The study was conducted according to the guidelines of the Declaration of Helsinki, and ethical review and approval were waived due to the retrospective design of the study.

Informed Consent Statement: Informed consent was obtained from all the subjects involved in the study.

Data Availability Statement: Data available on request from the corresponding author. The data are not publicy available due to restrictions for privacy.

Conflicts of Interest: The authors declare no conflict of interest.

\section{References}

1. Learmonth, I.D.; Young, C.; Rorabeck, C. The operation of the century: Total hip replacement. Lancet 2007, 370, 1508-1519. [CrossRef]

2. Brooks, P.J. Dislocation following total hip replacement: Causes and cures. Bone Jt. J. 2013, 95, 67-69. [CrossRef] [PubMed]

3. D'Angelo, F.; Zagra, L.; Moretti, B.; Virgilio, A.; Mazzacane, M.; Solarino, G. Retrospective multi-centre study on head adapters in partial revision hip arthroplasty. Hip Int. 2020, 30, 72-76. [CrossRef] [PubMed]

4. Jones, S.A. The prevention and treatment of dislocation following total hip arthroplasty: Efforts to date and future strategies. Hip Int. 2015, 25, 388-392. [CrossRef] [PubMed]

5. Noyer, D.; Caton, J.H. Once upon a time.... Dual mobility: History. Int. Orthop. 2017, 41, 611-618. [CrossRef] [PubMed]

6. Cuthbert, R.; Wong, J.; Mitchell, P.; Jaiswal, P.K. Dual mobility in primary total hip arthroplasty: Current concepts. EFORT Open Rev. 2019, 4, 640-646. [CrossRef] [PubMed]

7. Solarino, G.; Piazzolla, A.; Mori, C.M.; Moretti, L.; Patella, S.; Notarnicola, A. Alumina-on-alumina total hip replacement for femoral neck fracture in healthy patients. BMC Musculoskelet. Disord. 2011, 12, 32. [CrossRef]

8. Adam, P.; Philippe, R.; Ehlinger, M.; Roche, O.; Bonnomet, F.; Molé, D.; Fessy, M.H. Dual mobility cups hip arthroplasty as a treatment for displaced fracture of the femoral neck in the elderly. A prospective, systematic, multicenter study with specific focus on postoperative dislocation. Orthop. Traumatol. Surg. Res. 2012, 98, 296-300. [CrossRef]

9. De Martino, I.; D’Apolito, R.; Soranoglou, V.G.; Poultsides, L.A.; Sculco, P.K.; Sculco, T.P. Dislocation following total hip arthroplasty using dual mobility acetabular components: A systematic review. Bone Jt. J. 2017, 99, 18-24. [CrossRef]

10. Tristaino, V.; Lantieri, F.; Tornago, S.; Gramazio, M.; Carriere, E.; Camera, A. Effectiveness of psychological support in patients undergoing primary total hip or knee arthroplasty: A controlled cohort study. J. Orthop. Traumatol. 2016, 17, 137-147. [CrossRef]

11. Buller, L.T.; Best, M.J.; Klika, A.K.; Barsoum, W.K. The influence of psychiatric comorbidity on perioperative outcomes following primary total hip and knee arthroplasty; a 17-year analysis of the National Hospital Discharge Survey database. J Arthroplast. 2015, 30, 165-170. [CrossRef] [PubMed]

12. De Caro, M.F.; Vicenti, G.; Abate, A.; Picca, G.; Leoncini, V.; Lomuscio, M.; Casalino, A.; Solarino, G.; Moretti, B. Optimal improvement in function after total hip and knee replacement: How deep do you know your patient's mind? J. Biol. Regul. Homeost. Agents 2015, 29, 95-102. [PubMed] 
13. Toro, G.; Moretti, A.; Paoletta, M.; De Cicco, A.; Braile, A.; Panni, A.S. Neglected femoral neck fractures in cerebral palsy: A narrative review. EFORT Open Rev. 2020, 5, 58-64. [CrossRef] [PubMed]

14. Mori, C.M.; Vicenti, G.; Carrozzo, M.; Picca, G.; Bizzoca, D.; Leone, A.; Morizio, A.; Solarino, G.; Moretti, B. The fake unlocked femoral nail: A configuration to avoid in stable pertrochanteric femur fractures. Injury 2018, 49 (Suppl. 3), S32-S36. [CrossRef] [PubMed]

15. Caiaffa, V.; Vicenti, G.; Mori, C.M.; Panella, A.; Conserva, V.; Corina, G.; Scialpi, L.; Solarino, G.; Moretti, B. Unlocked versus dynamic and static distal locked femoral nails in stable and unstable intertrochanteric fractures. A prospective study. Injury 2018, 49 (Suppl. 3), S19-S25. [CrossRef]

16. D'Arrigo, C.; Perugia, D.; Carcangiu, A.; Monaco, E.; Speranza, A.; Ferretti, A. Hip arthroplasty for failed treatment of proximal femoral fractures. Int. Orthop. 2010, 34, 939-942. [CrossRef] [PubMed]

17. Cho, C.H.; Yoon, S.H.; Kim, S.Y. Better Functional Outcome of Salvage THA Than Bipolar Hemiarthroplasty for Failed Intertrochanteric Femur Fracture Fixation. Orthopedics 2010, 33, 721. [CrossRef]

18. Archibeck, M.J.; Carothers, J.T.; Tripuraneni, K.R.; White, R.E., Jr. Total Hip Arthroplasty After Failed Internal Fixation Of Proximal Femoral Fractures. J. Arthroplast. 2013, 28, 168-171. [CrossRef]

19. Pui, C.M.; Bostrom, M.P.; Westrich, G.H.; Della Valle, C.J.; Macaulay, W.; Mont, M.A.; Padgett, D.E. Increased complication rate following Conversion Total Hip Arthroplasty after cephalomedullary fixation for intertrochanteric hip fractures: A multi-center study. J. Arthroplast. 2013, 28, 45-47. [CrossRef]

20. Bedard, N.A.; Martin, C.T.; Slaven, S.E.; Pugely, A.J.; Mendoza-Lattes, S.A.; Callaghan, J.J. Abnormally High Dislocation Rates of Total Hip Arthroplasty After Spinal Deformity Surgery. J. Arthroplast. 2016, 31, 2884-2885. [CrossRef]

21. Salib, C.G.; Reina, N.; Perry, K.I.; Taunton, M.J.; Berry, D.J.; Abdel, M.P. Lumbar fusion involving the sacrum increases dislocation risk in primary total hip arthroplasty. Bone Jt. J. 2019, 101, 198-206. [CrossRef]

22. Piazzolla, A.; Solarino, G.; Bizzoca, D.; Montemurro, V.; Berjano, P.; Lamartina, C.; Martini, C.; Moretti, B. Spinopelvic parameter changes and low back pain improvement due to femoral neck anteversion in patients with severe unilateral primary hip osteoarthritis undergoing total hip replacement. Eur. Spine J. 2018, 27, 125-134. [CrossRef] [PubMed]

23. Mudrick, C.A.; Melvin, J.S.; Springer, B.D. Late posterior hip instability after lumbar spinopelvic fusion. Arthroplast. Today 2015, 1, 25-29. [CrossRef] [PubMed]

24. Vicenti, G.; Solarino, G.; Spinarelli, A.; Carrozzo, M.; Picca, G.; Maddalena, R.; Rifino, F.; Moretti, B. Restoring the femoral offset prevent early migration of the stem in total hip arthroplasty: An EBRA-FCA study. J. Biol. Regul. Homeost. Agents 2016, 30, 207-212. [PubMed]

25. Prudhon, J.L.; Verdier, R.; Caton, J.H. Low friction arthroplasty and dual mobility cup: A new gold standard. Int. Orthop. 2017, 41, 563-571. [CrossRef] [PubMed]

26. Chalmers, B.P.; Syku, M.; Sculco, T.P.; Jerabek, S.A.; Mayman, D.J.; Westrich, G.H. Dual-Mobility Constructs in Primary Total Hip Arthroplasty in High-Risk Patients With Spinal Fusions: Our Institutional Experience. Arthroplast. Today 2020, 6, 749-754. [CrossRef] [PubMed] 